\title{
ANOPHELES (CULICIDAE, ANOPHELINAE) E A MALÁRIA EM BURITICUPU-SANTA LUZIA, PRÉ-AMAZÔNIA MARANHENSE
}

\author{
José Manuel Macário Rebêlo, Antonio Rafael da Silva, \\ Luiz Alves Ferreira e José Augusto Vieira
}

\begin{abstract}
Neste trabalho, estudou-se as espécies do gênero Anopheles que se atraem por iscas bumanas na Região de Buriticupu, Santa Luzia, Estado do Maranbão. Foram encontradas 7 espécies do subgênero Nyssorhyncus A. (N) darlingi, o principal vetor da malária humana na região, foi a espécie mais freqüente (53,1\% da amostra total). Seguem na ordem de importância, A. (N) evansae (21.0\%), A. (N) triannulatus (17.4\%) $e$ A. (N) nuñeztovari (4,8\%). As demais espécies, A. (N) argyritarsis, A. (N) oswaldoi $e$ A. (N) rangeli representaram juntas $3,7 \%$ da amostra total. As visitas foram mais frequentes no extra (51,7\% da amostra total) do que no peridomicílio (45,7\%). A. (N) darlingi e A. (N) evansae foram as únicas espécies encontradas no intradomicílio, numa proporção de aproximadamente 2,6\% da amostra total. Os anofelinos ocorreram nas estações seca e chuvosa, com maior frequência na última, coincidindo com os elevados índices de caso de malária naquela região.
\end{abstract}

Palavras-chaves: Anopheles. Malária. Pré-Amazônia. Maranhão.

Os Anofelinos são um grupo de dípteros hematófagos com ampla distribuição mundial. O gênero Anopheles contém várias espécies vetoras de plasmódios da malária. No contexto mundial, entre os vetores mais importantes, se acham os grupos A. maculipennis (Europa), culicifacies (India), minimus (Assam a China e Filipinas), gambiae e funestus na África ${ }^{20}$.

No Neotrópico, A. albimanus (México), $A$. nuñeztovari (Venezuela e Colombia) ${ }^{11}{ }^{13}, A$. darlingi e A. aquasalis (Brasil) 2616119 , são bem conhecidas como vetores de plasmódios maláricos. Outras espécies importantes na transmissão da malária em nosso país são $A$. bellator e $A$. cruzzi $\imath^{3}$, os equivalente ecológicos das duas últimas, no sul do Brasil, região atualmente livre desta enfermidade. Menos importante é $A$. albitarsis, vetor potencial da malária nas áreas onde ocorre.

Departamento de Patologia. Núcleo de Patologia Tropical e Medicina Social. Universidade Federal do Maranhão, São Luiz, MA.

Projeto Buriticupu convênio Universidade Federal do Maranhão/MS/Fundação Nacional de Saúde.

Endereço para correspondência: Dr. José Manuel Macário Rebêlo. Dept ${ }^{0}$ de Patologia/UFMA. Pça Madre Deus 2, São Luís, MA. Tel: (098) 248-2429; Fax: (098) 2225135.

Recebido para publicação em 09/11/95.
No Maranhão, são duas as principais espécies vetores de plasmódios: darlingi, no interior, e aquasalis, no litoral ${ }^{515}{ }^{17}$. Outras espécies que integram a extensa fauna anofelina, e que ocorrem no nosso Estado, são eventualmente incriminadas como vetores secundários em outras áreas amazônicas ${ }^{61619}$.

Apesar do Maranhão ser conhecido como área endêmica da malária e de se fazer referência aos principais vetores aqui existentes, não existe nenhum trabalho científico, padronizado, com o objetivo de determinar a fauna anofelina local. Estudos de levantamentos da fauna de áreas malarígenas são importantes do ponto de vista epidemiológico, especialmente se essas áreas vêm sofrendo o impacto progressivo do desmatamento, como ocorre notadamente na região de Buriticupu, PréAmazônia maranhense, ou mesmo, na Ilha de São Luís, capital do Estado do Maranhão.

Apesar da situação atual da malária nessas regiões do Estado, o conhecimento da fauna anofelina do Maranhão deriva, tão somente, dos inquéritos entomológicos realizados pelos técnicos da Fundação Nacional de Saúde, usualmente no intra e peridomicílios, com o intuito de buscar meios de controle. Não tem havido uma preocupação no sentido de definir a fauna, quanto a diversidade, distribuição e aspectos ecológicos a partir dos habitats naturais. 
Rebêlo JMM, Silva AR, Ferreira LA, Vieira JA. Anopheles (Culicidae, anophelinae) e a malária em BuriticupuSanta Luzia, Pré-Amazônia maranhense. Revista da Sociedade Brasileira de Medicina Tropical 30:107-111, mar-abr, 1997.

Pretende-se, com este trabalho, definir as espécies de anofelinos que ocorrem em Buriticupu e que se associam com homem, determinando a densidade das populações, a ocorrência sazonal, a preferência por horário de atividade no intra, peri e extradomicílios.

\section{MATERIAL E MÉTODOS}

Área de estudo. A Região de Buriticupu encontra-se no Município de Santa Luzia, Estado do Maranhão, entre $4^{\circ}$ e $5^{\circ} L S$ e $45^{\circ} 30^{\prime}$ e $47^{\circ} \mathrm{LW}$, na Região Amazônica que abrange as terras maranhenses a oeste do Meridiano $44^{\circ}$. A altitude média é de $200 \mathrm{~m}$ acima do nível do mar. O relêvo deriva da Formação Itapecuru, do Cretáceo Inferior, com afloramento de tabuleiros isolados da Formação Barreira, do Terciário ${ }^{12}$.

O solo da região é o latossolo amarelo, antigamente coberto por Floresta Estacional Perenifólia Densa, com dossel mais denso e fechado do que a Floresta Estacional Aberta, a qual aparecia como manchas em alguns pontos. A vegetação original vem sendo devastada pela atividade madereira, projetos agropecuários e, em menor escala, pelo assentamento de colonos. Atualmente o remanescente da vegetação original é representada por glebas de matas isoladas sobre alguns platôs ${ }^{12}$.

A região está subordinada ao clima quenteúmido de transição entre o superúmido amazônico e o semi-árido nordestino, com 6 meses secos. A precipitação média anual é de $1800 \mathrm{~mm}$.

A área de estudo propriamente dita situa-se no povoado de Buritizinho, em Buriticupu, às margens da BR-222 que liga Santa Luzia a Açailândia e é cortada pelo Rio Buriticupu, afluente do Pindaré, o principal rio da região e um dos formadores da bacia do Mearim. As margens do Rio Buritizinho, outrora cobertas por matas ciliares, sofreu processo intenso de desmatamento nos últimos anos, contribuindo para a elevação do índice de evaporação especialmente no período de estiagem.

Amostragem. Os anofelinos foram estudados durante cinco meses: outubro e dezembro de 1994 e março, maio e setembro de 1995 . O método básico utilizado, foi a captura de fêmeas em iscas humanas, com uso de tubo de sucção. As capturas foram realizadas por apenas um coletor no extra, peri e intradomićlios, nos horários das 17:30 às 20:00 horas, durante 3 dias consecutivos. Os exemplares capturados foram transportados para o Posto de Saúde da UFMA, em Buriticupu, e identificados pelos técnicos da FNS.

\section{RESULTADOS}

Foram coletados 269 espécimens de 7 espécies de Anopheles, todas pertencentes ao subgênero Nyssorbyncus, a saber: Anopheles ( $N$ ) argyritarsis Robineau Desvoidy, 1827, Anopheles (N) darlingi Root, 1926, Anopheles (N) evansae (Brethes, 1926), Anopheles (N) nuñeztovari Gabaldón, 1940, Anopheles (N) oswaldoi (Peryassu, 1922), Anopheles (N) rangeli Gabaldón, Cova-Garcia \& Lopez, 1940 e Anopheles (N.) triannulatus (Neiva \& Pinto, 1922).

A. darlingi foi a espécie mais frequente na área de estudo, sendo representada por $53,1 \%$ da amostra total. Seguem-se na ordem de importância, A. evansae (21,0\%), A. triannulatus $(17,4 \%)$ e $A$. nuñeztovari (4,8\%). As demais espécies, $A$. argyritarsis, $A$. oswaldoi e $A$. rangeli, representaram juntas $3,7 \%$ da amostra total (Tabela 1).

Tabela 1 - Número de fêmeas de Anopheles capturadas em iscas bumanas, no extra, peri e intradomicílios, em Buriticupu, Santa Luzia, $M A$

\begin{tabular}{lrrrrr}
\hline Espécies de & \multicolumn{3}{c}{ Domicílios } & & \\
\cline { 2 - 4 } Anopheles & extra & peri & intra & Total & $\%$ \\
\hline A. argyritarsis & 1 & 1 & & 2 & 0,7 \\
A. darlingi & 86 & 52 & 5 & 143 & 53,1 \\
A. evansae & 28 & 26 & 2 & 56 & 21,0 \\
A. oswaldoi & 1 & 3 & & 4 & 1,5 \\
A. nuñeztovari & 8 & 5 & & 13 & 4,8 \\
A. rangeli & 2 & 2 & & 4 & 1,5 \\
A. triannulatus & 13 & 34 & & 47 & 17,4 \\
\hline Total/\% & $139 / 51,7$ & $123 / 45,7$ & $7 / 2,6$ & $269 / 100,0$ & 100,0
\end{tabular}

As atrações dos anofelinos às iscas foram mais frequentes no extra $(51,7 \%$ da amostra total) do que no peridomicílio (45,7\%), embora estatisticamente não tenha existido diferenças significativas na frequência das fêmeas entre estes dois ambientes ( $p>0,01)$. O intradomicílio foi pouco freqüentado e, por apenas 2,6\% dos indivíduos capturados pertencentes à duas espécies, A. darlingi e A. evansae (Tabela 1).

As primeiras visitas às iscas para o repasto sanguíneo ocorreram próximo das 18:00 horas, sendo mais intenso entre $18-19$ horas $(68,7 \%$ das fêmeas amostradas), diminuindo próximo das 19:30 horas até cessar após as 20:00 horas. Duas espécies, $A$. darlingi e $A$. evansae, foram 
Rebêlo JMM, Silva AR, Ferreira LA, Vieira JA. Anopheles (Culicidae, anophelinae) e a malária em BuriticupuSanta Luzia, Pré-Amazônia maranhense. Revista da Sociedade Brasileira de Medicina Tropical 30:107-111, mar-abr, 1997.

encontradas das 17:30-20:00h; as outras restringiram suas atividades a um período mais curto (Tabela 2).

\begin{tabular}{|c|c|c|c|c|c|}
\hline \multirow{2}{*}{$\begin{array}{l}\text { Espécies de } \\
\text { Anopheles }\end{array}$} & \multicolumn{5}{|c|}{ Horários } \\
\hline & $17: 30-18: 00$ & 18:00-18:30 & $18: 30-19: 00$ & $19: 00-19: 30$ & 19:30-20:00 \\
\hline A. argyritarsis & & & & & 2 \\
\hline A. darling $i$ & 3 & 53 & 43 & 27 & 17 \\
\hline A. evansae & 1 & 17 & 20 & 14 & 4 \\
\hline A. oswaldo $i$ & & 2 & 1 & 1 & \\
\hline A. nuñeztovari & & 7 & 2 & 4 & \\
\hline A. rangeli & & 1 & 2 & 1 & \\
\hline A. triannulatus & & 14 & 23 & 8 & 2 \\
\hline Total $/ \%$ & $4 / 1,5$ & $94 / 34,9$ & $91 / 33,8$ & $55 / 20,5$ & $25 / 9,3$ \\
\hline
\end{tabular}

A maior abundância de anofelinos em atividade hematofágica ocorreu nos meses da estação úmida, março $(37,5 \%)$ e maio $(29,4 \%)$. Todas as espécies foram registradas nas duas estações, mas A. triannulatus foi a única encontrada em todos os meses estudados, sendo mais frequente em dezembro e março. $A$. darlingi não foi atraída em dezembro e o pico de atividade ocorreu em março. $A$. evansae e $A$. nuñeztovari foram encontradas no mesmo período (março, maio e setembro), $A$. rangeli só ocorreu nos meses chuvosos, $A$. oswaldo $i$, nos meses de outubro e dezembro e A. argyritarsis, dezembro e março (Tabela 3 ).

Tabela 3 - Número de fêmeas de Anopheles capturadas em iscasbumanas, de acordo com o período do ano, em Buriticupu, Santa bumanas, de acordo com o periodo do ano, em Buriticupu, Santa
Luzia, MA.

\begin{tabular}{lccccc}
\hline & \multicolumn{5}{c}{ Meses } \\
\cline { 2 - 6 } Anopheles & outubro dezembro & março & maio & setembro \\
\hline A. argyritarsis & & 1 & 1 & & \\
A. darlingi & 8 & 38 & 54 & 39 & 4 \\
A. evansae & & & 24 & 26 & 6 \\
A. oswaldoi & 1 & 3 & & & \\
A. nuñeztovari & & & 2 & 7 & 4 \\
A. rangeli & & & 2 & 2 & \\
A. triannulatus & 5 & 16 & 18 & 5 & 3 \\
\hline Total & 14 & 58 & 101 & 79 & 17
\end{tabular}

\section{DISCUSSÃO}

Neste estudo, foi dado ênfase a um programa destinado a evidenciar a diversidade de espécies de Anopheles de importância médica. Portanto, foram incluídas no estudo, apenas as espécies de hábitos secundariamente antropófilos e que se aproximavam das habitações humanas, atraídas por seus moradores e sobre os quais se alimentavam. As espécies de hábitos essencialmente silvestres e zoófilas ficaram de fora da análise. Estas serão estudadas posteriormente.

A despeito do que foi exposto, encontrouse uma fauna variada com elementos de padrão de distribuição diverso, comuns às diferentes áreas biogeográficas do neotrópico, de hábito variável, com maior ou menor grau de antropofila. Algumas espécies encontradas em Buriticupu se caracterizaram como típicas de zonas quentes e úmidas, de ambientes amazônicos, pois são as mesmas que predominam em outras localidades de semelhante clima, como no Estado do Pará ${ }^{19}$. Além das espécies encontradas neste estudo, tem-se conhecimento da existência de pelo menos mais três que ocorrem na Pré-Amazônia maranhense (região de Imperatriz) e que não foram registradas na área de estudo, em Buriticupu. Entre elas pode-se citar $A$. albitarsis, a espécie mais importante naquela região, depois de $A$. darlingi $^{18}$, e $A$. galvaoi e A. strodei. Estas duas últimas espécies são pouco frequentes nas áreas urbanas.

Entre as espécies encontradas em Buriticupu, $A$. darlingi continua a ser a que se apresenta em maior densidade corroborando com as observações prévias de Silva ${ }^{17}$; é uma espécie muito comum nas zonas interioranas das Américas do Sul e Central.

Em Buriticupu, durante o período de desenvolvimento deste estudo, a malária alcançou índices elevados (1.260 casos), especialmente nos meses chuvosos. Neste mesmo período observou-se os maiores picos na atividade hematofágica de $A$. darlingi, o mais importante transmissor da malária no interior do Maranhão.

A. evansae também ocorreu em alta densidade, sendo a segunda espécie mais abundante no extra e peridomicílios. No interior das casas foi encontrada em baixa densidade. É uma espécie neotropical e considerada zoófila e pouco domiciliar em alguns locais, enquanto em outros tem se mostrado altamente domiciliar. É conhecida por albergar oocisto no estômago ${ }^{3}$ e, por esse motivo, pode desempenhar papel vetorial secundário, porém, em locais onde $A$. darlingi seja incomum.

Em Buriticupu, a malária tem causado sérias preocupações, pois, além de se constituir na principal endemia da região, seu processo de transmissão vem ocorrendo, ao que parece, mais no extra ou peridomicílio do que propriamente no interior das $\operatorname{casas}^{17}$. Nossas 
Rebêlo JMM, Silva AR, Ferreira LA, Vieira JA. Anopheles (Culicidae, anophelinae) e a malária em BuriticupuSanta Luzia, Pré-Amazônia maranhense. Revista da Sociedade Brasileira de Medicina Tropical 30:107-111, mar-abr, 1997.

observações mostraram maior frequência de atividade hematofágica dos anofelinos, no extra e peridomicílio do que no intradomicílio. É válido ressaltar que apesar de não termos realizado observações no crepúsculo matutino, a baixa freqüência e/ou a ausência de $A$. darlingi no intradomicílio no crepúsculo vespertino/primeiras horas da noite, constituise um grande problema porque pode dificultar as estratégias de combate à malária.

A exofilia de $A$. darlingi é conhecida desde alguns anos na Amazônia brasileira 26161 19; comportamento similar se verifica para A. funestus no oeste africano ${ }^{20}, A$. albimanus no México e $A$. nuñeztovari na Venezuela e Colombia $^{11} 13$

É digno ressaltar o papel importante que $A$. nuñeztovari desempenha na transmissão da malária no oeste da Venezuela e norte da Colômbia ${ }^{10}$. Nessas áreas ela prefere os horários tardios da noite, enquanto no Brasil ocorre no crepúsculo vespertino/primeiras horas da noite ${ }^{19}$. Buriticupu não foi exceção deste padrão. Naquela localidade $A$. nuñeztovari não é muito comum e tem sido encontrada fora das habitações e em baixa densidade. Contudo, na vizinha região de Tucuruí/Marabá, Estado do Pará, ocorre em alta densidade populacional e apresenta acentuada antropofilia. Estes fatores levaram ${ }^{19}$ a considerar a possibilidade desta espécie atuar como vetor secundário nesta última região.

Na Amazônia, foi questionado também o papel secundário que $A$. oswaldo $i$ e $A$. triannulatus desempenham como vetores da malária' ${ }^{19}$. Essas espécies, como A. nuñeztovari, coexistem em Buriticupu, porém em freqüência bem mais baixa do que $A$. darlingi e A. evansae. Os dados disponíveis não permitem aceitar ou refutar a possibilidade de que elas possam apresentar algum papel na transmissão da malária em Buriticupu.

A. oswaldoi era tida como uma espécie típica da zona litorânea ${ }^{14}$ porém é comum e amplamente distribuída no interior do Pará ${ }^{19} \mathrm{e}$ tem sido registrada em Buriticupu, inclusive este não é o primeiro registro para aquela região ${ }^{17}$. Em regra é considerada essencialmente zoófila e, apesar de infectar-se, em condições experimentais, com Plasmodium vivax e $P$. falciparum, não desempenha papel de importância na transmissão da malária, pois não tem sido encontrada naturalmente infectada ${ }^{48}$.
Quanto à $A$. argyritarsis, é neotropical e, apesar de não ter sido até o momento incriminada na transmissão da malária ${ }^{4514}$, ela pica o homem e é encontrada próximo às habitações. Em Buriticupu foi encontrada em baixa densidade. Comportamento similar tem sido observado no município de Imperatriz nos últimos dois anos ${ }^{18}$. Vários autores ${ }^{14}$ afirmam que embora possa ser encontrada nas casas, não é verdadeiramente domiciliar e mostra grande indiferença pelo sangue humano. O único exemplar capturado no estudo corrobora essas observações.

Os dados encontrados, se por um lado, indicam a existência de uma fauna anofelina variada, por outro, são preocupantes, pois, a presença de $A$. darling $i$ no extra, peri e intradomicílio, e a característica endêmica da malária em Buriticupu, indicam que a sua transmissão pode ocorrer, potencialmente, nos três ambientes. Ressalta-se, porém, que a prevalência do repasto sanguíneo fora das habitações é o maior indicativo de que a transmissão da malária em Buriticupu vem se processando, principalmente no ambiente extradomiciliar.

\section{SUMMARY}

Female anophelines were captured in buman baits in the region of Buriticupu, Santa Luzia, Maranhão State. Seven species belonging the subgenus Nyssorhyncus were found. Anopheles (N.) darlingi, the principal vector of human malaria, was the most abundant (53.1\%) followed by A. (N.) evansae (21.0\%), A. (N.) triannulatus (17.4\%) e A. (N.) nuñeztovari (4.8\%). The others, A. (N.) argyritarsis, A. (N.) oswaldoi and A. (N.) rangeli, were less frequently found, representing only 3.7\% of the total sample. The anophelines were most frequent in both the extra (51.7\%) and peridomiciles (45.7\%). The intradomicile was visited by some specimens of the A. (N.) darlingi and A. (N.) evansae (active in both the rain and dry seasons, especially in the former, when the malaria reached high leves of transmission.

Key-words: Anophelines. Malaria. Amazônia. Maranhão.

\section{REFERÊNCIAS BIBLIOGRÁFICAS}

1. Albuquerquer C. Foco residual de malária na Rodovia Manaus-Boa Vista. Tese de Mestrado. Universidade Federal do Rio de Janeiro, Rio de Janeiro, RJ, 1982. 
Rebêlo JMM, Silva AR, Ferreira LA, Vieira JA. Anopheles (Culicidae, anophelinae) e a malária em BuriticupuSanta Luzia, Pré-Amazônia maranhense. Revista da Sociedade Brasileira de Medicina Tropical 30:107-111, mar-abr, 1997.

2. Alecrim WD. Estudos clínicos e epidemiológicos da malária no Rio Amazonas. Brasília. Tese de Mestrado. Faculdade de Ciências Médicas da Universidade de Brasília, Brasília, DF, 1979.

3. Correa R, Ramos AS. Os anofelinos da região meridional do Estado de São Paulo. Arquivos de Higiene e Saúde Pública 7: 37-57, 1942.

4. Coutinho JO. Contribuição para o estudo da distribuição geográfica dos anofelinos do Brasil. Tese de Livre Docência. Universidade de São Paulo, São Paulo, SP, 1947.

5. Deane LM, Causey SR, Deane MP. Notas sobre a distribuição e a biologia dos anofelinos das regiões nordestinas e amazônicas do Brasil. Revista do Serviço Especializado em Saúde Pública 1: 827-934, 1948.

6. Dixon KE, Roberts DR, Llewellyn CH. Contribuição ao estudo epidemiológico da malária em trecho da Rodovia Transamazônica, Brasil. Revista do Instituto de. Medicina. Tropical São Paulo 21:287-292, 1979.

7. Elliott R. Studies on man-vector contact in some malarious areas in Colombia. Bulletin of the World Health. Organization 38:239-253, 1968.

8. Faran ME, Linthicum KJ.A handbook of the Amazonian species of Anopheles (Nyssorbyncus) (Diptera, Culicidae). Mosquito Systematics 13:0191,1981

9. Ferreira E. Distribuição geográfica dos anofelinos no Brasil e sua relação com o estado atual da erradicação da malária. Revista Brasileira de Malariologia e Doenças Tropicais 16:329-348, 1964.

10. Gabaldon A, Guerreiro L, Martin GG. Malária refractária en el occidente de Venezuela. Revista Venezolana Sanidad 14:513-530, 1963.

11. Garcia-Martins G. Status of malaria eradication in the Americas. The American Journal Tropical Medicine and Hygiene 21:617-23, 1972.
12. Instituto Brasileiro de Geografia e Estatística.Atlas do Maranhão. Edição em convênio com o governo do Estado do Maranhão. Rio de Janeiro, 104 p. 1984.

13. Liu SY. Problemas asociados com el vetor: resistencia fisiologica y compledos de especies. In: Malaria en las Americas. Washington, Publicações Científicas 405, 1981.

14. Pessoa SB, Martins AV. Parasitologia Médica. $10^{\text {a }}$ edição. Guanabara Koogan, Rio de Janeiro, RJ, 1978.

15. Rachou RG.Anofelinos do Brasil: comportamento das espécies vetoras da Malária. Revista Brasileira de Malariologia e Doenças Tropicais 10:145-181, 1958.

16. Roberts DR, Hoch AL, Peterson ME, Pinheiro FP. Programa multidisciplinario de vigilância de las enfermidades infecciosas en zonas colindantes con la carretera transamazonica en Brasil. IV. Estudio Entomologico. Boletim Oficina Sanitaria Panamá 91:379-398, 1981.

17. Silva AR. Malária: fotografia de uma crise no setor saúde. Universidade Federal do Maranhão, São Luís, MA, 1989.

18. Sousa JRP. Contribuição ao estudo epidemiológico da malária no município de Imperatriz, Estado do Maranhão. Monografia apresentada ao Curso de Ciências Habilitação em Biologia. Imperatriz, MA. 1995.

19. Tadei WP, Mascarenhas BM, Podestá MG. Biologia de anofelinos amazônicos. VIII. Conhecimentos sobre a distribuição de espécies de Anopheles na região de Tucuruí-Marabá (Pará). Acta Amazônica 13:103-140, 1983.

20. Zahar AP.Vector control operations in the African Contex. Bulletin of the World Health Organization 62 (supl):89-100, 1984. 